\title{
GNRH1 Variants in Congenital Hypogonadotropic Hypogonadism: Single-Center Experience and Systematic Literature Review
}

\author{
Virendra A. Patil ${ }^{a} \quad$ Anurag Ranjan Lila ${ }^{a}$ Nalini Shah ${ }^{a} \quad$ Alka V. Ekbote ${ }^{b}$ \\ Ravikumar Shah ${ }^{a}$ Vishwambhar Vishnu Bhandarec Vijaya Sarathi $^{d}$ \\ Sneha Arya ${ }^{a}$ Saba Samad Memon ${ }^{a}$ Ambarish Kunwar ${ }^{c}$ Tushar Bandgar ${ }^{a}$ \\ aDepartment of Endocrinology, Seth G S Medical College and KEM Hospital, Mumbai, India; ${ }^{b}$ Clinical and Molecular \\ Genetics, Kamalnayan Bajaj Hospital, Aurangabad, India; ' Department of Biosciences and Bioengineering, Indian

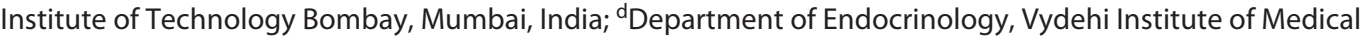 \\ Sciences and Research Centre, Bengaluru, India
}

\section{Keywords}

Congenital hypogonadotropic hypogonadism · GNRH1

\begin{abstract}
Objective: As GNRH1 genotype-phenotype correlation in $\mathrm{CHH}$ is not well studied, we aim to describe the GNRH1 variants in our $\mathrm{CHH}$ cohort and present a systematic review as well as genotype-phenotype analysis of all mutation-positive cases reported in the world literature. Design: This is a retrospective study of GNRH1 mutation-positive patients from a western Indian center. PRISMA guidelines-based PubMed search of the published literature of all GNRH1 mutation-positive patients was conducted. Setting: This study was conducted in an academic medical center. Patient(s): This study included 2 probands from our cohort and 19 probands from the world literature. Main Outcome Measure(s): Demographic details, clinical presentation, biochemistry, imaging, treatment details, and genotypic data were recorded. Result(s): Two probands in our cohort carried two novel pathogenic biallelic GNRH1 variants (p.Glu24Leu, c.238$2 A>G$ ). Both had a severe reproductive phenotype. We report successful gonadotropin therapy and fertility in 1 proband. We included 19 probands from 12 studies after the
\end{abstract}

literature review. Ten $\mathrm{CHH}$ probands (inclusive 2 from this study) with biallelic GNRH1 variants had severe reproductive phenotype, low gonadotropin levels, low/normal prolactin, normal pituitary imaging, and no extra-reproductive phenotype. Of seven biallelic variants reported, three were frameshift, two were splice-site, and two were missense mutations. All of them were pathogenic/likely pathogenic without oligogenicity. Of seven monoallelic GNRH1 variants reported in 11 probands, 4 had nonreproductive phenotype, 3 were benign/likely benign, and 4 were oligogenic. Conclusion(s): GNRH1 biallelic variants lead to severe reproductive phenotype, with low gonadotropin levels without nonreproductive features or oligogenicity. However, the role of GNRH1 monoallelic variants in $\mathrm{CHH}$ pathophysiology for reported variants remains questionable.

(c) 2021 S. Karger AG, Basel

\section{Introduction}

Congenital hypogonadotropic hypogonadism $(\mathrm{CHH})$ is a genetically heterogeneous disorder with more than forty genes implicated in its pathogenesis [1]. The role of gonadotropin-releasing hormone $(\mathrm{GnRH})$ in $\mathrm{CHH}$ was

Correspondence to:

Anurag Ranjan Lila, anuraglila@gmail.com 
demonstrated in 1986 when a 33.5-kb deletion in GNRH was identified in $h p g$ mice (naturally occurring hypogonadal mice) [2]. Its role was further validated with the report of reversal of phenotype after effective gene therapy [3]. Although GNRH1 was the most obvious candidate gene for $\mathrm{CHH}$, it took over two decades to find the first human case of $\mathrm{CHH}$ due to a pathogenic variant [4].

GNRH1 is located on 8p21.2 and comprises four exons and three introns. Exon 1 contains the $5^{\prime}$-untranslated region ( $5^{\prime}$-UTR). Exon 2 encodes part of the prepro$\mathrm{GnRH}$ polypeptide, consisting of a signal peptide, $\mathrm{GnRH}$ decapeptide, Gly-Lys-Arg cleavage site, and 23 amino acids of GnRH-associated peptide (GAP). Exon 3 encodes GAP's middle 32 amino acids, whereas exon 4 encodes GAP's last 13 amino acids and 3'-UTR [5]. Exons 3 and 4 also have elements of exonic splicing enhancers (ESEs) for pre-mRNA splicing as shown in in vitro studies $[6,7]$. The length of GnRH1 has been evolutionally conserved, and specific residues (positions 1, 4, 9, and 10) are identical amongst vertebrates [8]. To date, only a few cases of $\mathrm{CHH}$ with GNRH1 variants have been reported worldwide. Most of them harbor biallelic variants with an autosomal recessive mode of inheritance [4, 9-14]. Monoallelic variants with or without oligogenicity have also been reported [9, 15-19]. An effort to demonstrate genotypephenotype correlation with the hypothesis of a dominant-negative role of the most common reported monoallelic variant (p.Arg31Cys) was unsuccessful [20]. Genotype-phenotype data in $\mathrm{CHH}$ patients with GNRH1 variants have not been studied systematically. Analyzing the clinical, biochemical, and genetic data of such a rare condition may further our understanding of the basic physiology of the hypothalamo-pituitary-gonadal axis.

Here, we describe 2 probands with biallelic GNRH1 pathogenic variants from a large Asian Indian $\mathrm{CHH}$ cohort. Additionally, a systematic literature review of the reported $\mathrm{CHH}$ patients harboring GNRH1 variants published to date was done.

\section{Methods}

The present study is part of the research project No.EC/159/2009 entitled "Genotype, Phenotype and Radiological Correlation of Idiopathic Hypogonadotropic Hypogonadism," approved by the Institutional Ethics Committee II of Seth GS Medical College, Mumbai; $\mathrm{CHH}$ index patients registered between January 2010 and January 2018 were included in the study. Inclusion and diagnostic criteria for $\mathrm{CHH}$ were described previously [21]. Genetic analysis was done after obtaining written informed consent. Wherever available, segregation studies were performed for the observed variant is available and alive family members of the index cases.
Demographic profile, clinical findings, hormonal profile, MRI of the pituitary, and treatment outcomes of $\mathrm{CHH}$ patients with GNRH1 variants were recorded. Baseline reproductive phenotype was considered to be severe if a testicular volume was less than 4 $\mathrm{mL}$ in males and absent breast development in females, whereas a testicular volume $\geq 4 \mathrm{~mL}$ in males and some breast development in females was considered as partial reproductive phenotype [22].

Serum follicle-stimulating hormone (FSH), luteinizing hormone $(\mathrm{LH})$, total testosterone $(\mathrm{T})$, and prolactin levels were measured by chemiluminescence immunoassay (ADVIA Centaur XP Immunoassay Systems; Siemens). Intraassay and interassay coefficients of variation were less than $10 \%$ for all the hormonal assays. Lower limits of detection for $\mathrm{FSH}, \mathrm{LH}, \mathrm{T}$, and prolactin were 0.3 $\mathrm{mIU} / \mathrm{mL}, 0.07 \mathrm{mIU} / \mathrm{mL}, 0.05 \mathrm{ng} / \mathrm{dL}$, and $0.3 \mathrm{ng} / \mathrm{mL}$, respectively.

Clinical exome sequencing was carried out using Illumina's Sequencing. This clinical exome panel consisted of 4,811 genes which had 29 known $\mathrm{CHH}$ genes. We present data of GNRH1 variants. The targeted sequencing depth was 100X for GNRH1. The GATK best practices framework was followed to identify variants in the sample using Sentieon (v201808.01). The variants found were confirmed with Sanger sequencing. The functional implication of variants was predicted using in silico tools (Polyphen-2, Sort Intolerant from Tolerant, and MutationTaster, NNSplice, NetGene2). The minor allele frequency for the variants was checked in the databases like 1,000 Genomes and gnomAD.

\section{GnRH Decapeptide Structure-Function Prediction Model}

The full-length three-dimensional structure of the human GnRH receptor (GnRHR) was generated by homology modeling, using a template model generated by I-Tasser (https://zhanglab. dcmb.med.umich.edu/I-TASSER/). Structural refinement was done using steepest descent and conjugate gradient methods, and its quality check was done using the Ramachandran plot (outlier: $0.7 \%)$. The GnRH1 structure was retrieved from the RCSB structural database (PDB ID: 4D5M). The variant GnRH1 (p.Gln24Leu) structure was generated using the Chimera tool (https://www.cgl. ucsf.edu/chimera/). Docking of GnRHR with wild-type GnRH1 and its variants was done using HADDOCK 2.0 webserver (https:// www.bonvinlab.org/software/haddock2.2). Best-docked conformations obtained were further subjected for molecular dynamics (MD) simulation for $100 \mathrm{~ns}$ in the lipid bilayer using Gromacs 2018.0 software (http://www.gromacs.org/). The parameters for MD simulations were taken from an earlier reported similar study [23]. The stable trajectory was chosen for binding energy calculation using the MMPBSA approach (https://github.com/ RashmiKumari/g_mmpbsa).

\section{Literature Review}

A systematic review of the literature was performed as per Preferred Reporting Items for Systematic Review and Meta-Analyses guidelines [24] done by 2 authors together. The PubMed database (http://www.ncbi.nlm.nih.gov/pubmed) was searched on 20 April 2021 using the following search items: "Congenital Hypogonadotropic Hypogonadism" [All Fields] OR "Idiopathic Hypogonadotropic Hypogonadism" [All Fields] OR "GNRH1" [All Fields]. Studies describing $\mathrm{CHH}$ patients with biallelic and monoallelic GNRH1 variants were included for analysis. Cross-references of selected publications and review articles were used to find additional studies. The PubMed search yielded 2,256 articles. After exclusions (as detailed in Fig. 1), 12 studies were included in the final 


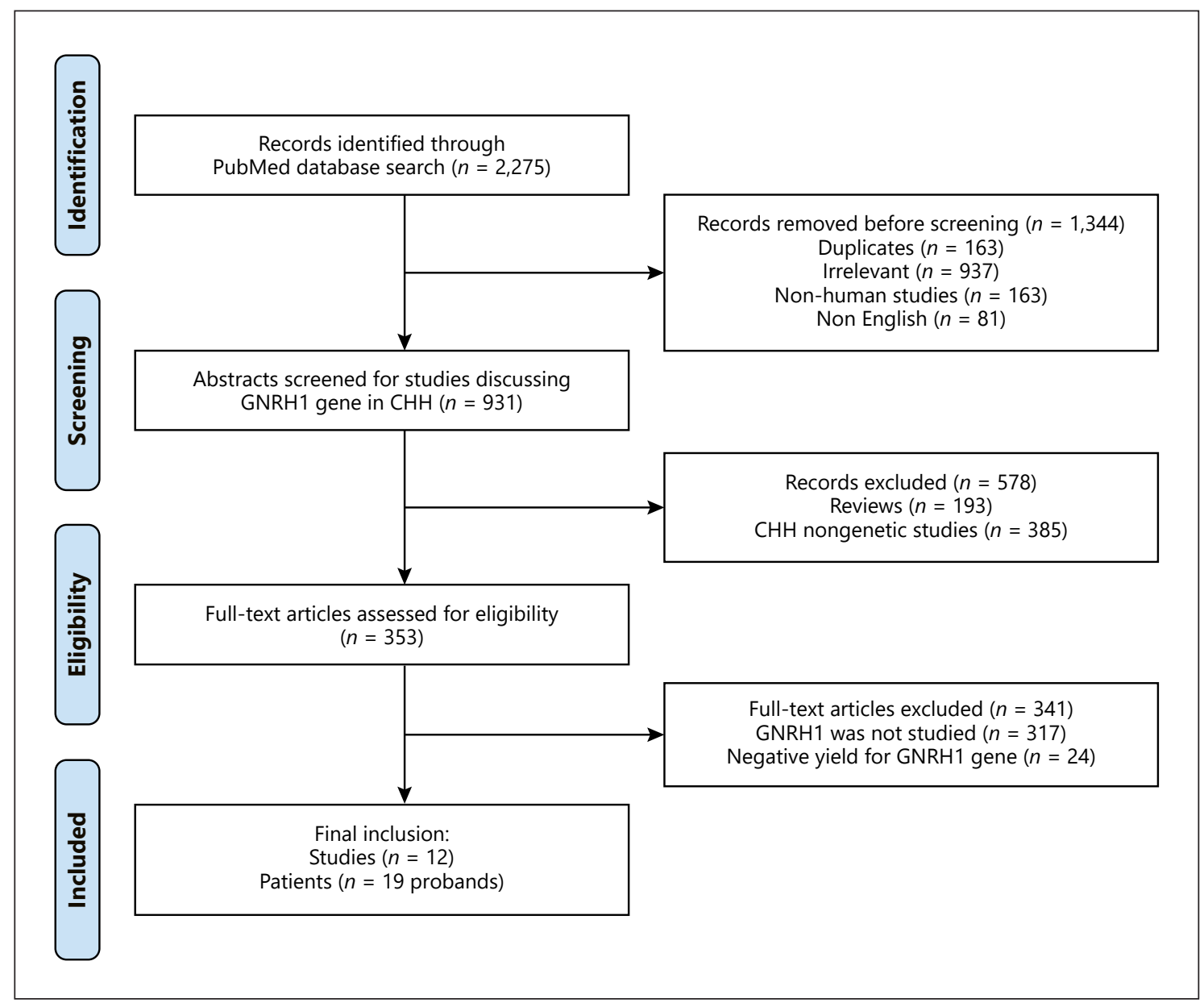

Fig. 1. The PRISMA flow diagram for the systematic review.

analysis yielding a cohort of 19 probands $(4,14-24)$. For the reported probands and their affected family members, demographic and phenotypic details, genetic variants, and treatment outcomes were recorded. The review was not registered. The VarSome prediction tool (http://varsome.com) [25] was used to classify all the reported variants as per ACMG variant classification guidelines [26].

\section{Results}

The prevalence of homozygous GNRH1 pathogenic variants in our $\mathrm{CHH}$ cohort was $1.3 \%(2 / 150)$. It was $2.9 \%$ (2/68) for normosmic $\mathrm{CHH}(\mathrm{nCHH})$ patients. Clinical details of the 2 patients from our center are as follows.

\section{Case 9}

A boy (born of nonconsanguineous marriage) presented at 15 years of age with small-sized genitalia and lack of other secondary sexual characteristics. He had normal olfaction, hearing, and no obvious nonreproductive phenotypic defects. He had micropenis (stretched penile length [SPL]: $3 \mathrm{~cm}$ ), bilateral testicular volumes of 1 $\mathrm{mL}$ each, Tanner's public hair stage 2 (P2), and no gynecomastia. His serum FSH, LH, and T levels were 1.56 $\mathrm{mIU} / \mathrm{mL}, 0.09 \mathrm{mIU} / \mathrm{mL}$, and $0.4 \mathrm{ng} / \mathrm{mL}$, respectively. $\mathrm{He}$ was started on T replacement therapy. At the last followup, at 18 years of age, his height was $169 \mathrm{~cm}(-0.7$ SDS $)$ with an arm span of $182 \mathrm{~cm}$. His mother had attained menarche at 13 years of age; she reported having had spontaneous regular menses, unassisted conception, and two normal pregnancies. The father also had normal pubertal development. His 15-year-old younger brother lacks secondary sexual characters but has denied clinical and biochemical evaluation.

A novel splice-acceptor variant, c.238-2A $>\mathrm{G}$, at intron 3 -exon 4 junction was identified in a homozygous state in 
the GNRH1 gene in the proband and his younger sibling; both parents were carriers. The VarSome prediction tool classified c.238-2A $>\mathrm{G}$ as pathogenic (P). No variants were found in the other known $\mathrm{CHH}$ genes $(n=28)$.

\section{Case 10}

A 25-year-old male (born of consanguineous marriage) presented with small-sized genitalia and a lack of secondary sexual characteristics. His height was $164 \mathrm{~cm}$ $(-1.4$ SDS), with an arm span of $177 \mathrm{~cm}$. He had micropenis (SPL: $3.5 \mathrm{~cm}$ ), bilateral small testes $(1 \mathrm{~mL})$ with P2, but no gynecomastia. He had normal olfaction, hearing, and no obvious nonreproductive phenotypic defects. His serum FSH, $\mathrm{LH}$, and $\mathrm{T}$ levels were $0.5 \mathrm{mIU} / \mathrm{mL},<0.1$ $\mathrm{mIU} / \mathrm{mL}$, and $0.15 \mathrm{ng} / \mathrm{mL}$, respectively. His MRI brain revealed normal-sized olfactory bulbs and normal pituitary morphology. As he desired fertility, he was started on gonadotropin therapy with objectives of both pubertal induction and fertility. He was started on injection human menopausal gonadotropin (hMG) 150 units subcutaneously thrice a week and human chorionic gonadotropin (hCG) 1,000 units subcutaneously twice a week, which was increased to 2,000 units thrice a week after 6 months. His testicular volume increased from $1 \mathrm{~mL}$ to 8 $\mathrm{mL}$ each after 18 months of continued treatment. Semen analysis showed azoospermia even after 12 months of treatment, but with continued treatment, sperm count rose to $32 \mathrm{million} / \mathrm{mL}$ ( $50 \%$ forward progression) at 18 months after treatment. He fathered a child naturally. He had a novel missense variant (p.Gln24Leu) in the homozygous state in the GNRH1 gene, and his parents were carriers of the same variant in the heterozygous state. The VarSome prediction tool classified this variant as likely pathogenic (LP). No variants were observed in other known $\mathrm{CHH}$ genes $(n=28)$. Figure 2 shows the MD simulation end structure of GnRHR (shown in gold) with wild-type GnRH1 (a) and the mutant, GnRH1 (p.Gln24Leu) (b). Binding affinity for mutant GnRH1 (p.Gln24Leu) is less due to poor interactions observed at the transmembrane helices at the outer surface. These findings are further supported by the calculated binding energy over stable MD simulation trajectory using the MMPBSA approach. The binding affinity of GnRHRGNRH1 (p.Gln24Leu) $(-91.68 \pm 11.19 \mathrm{kcal} / \mathrm{mol})$ was less than that of GnRHR-GNRH1 wild-type $(-151.15 \pm 8.46$ $\mathrm{kcal} / \mathrm{mol})$.

\section{Phenotype-Genotype Characteristics}

A total of 19 probands harboring GNRH1 variants (8 biallelic and 11 monoallelic) were identified in the litera-

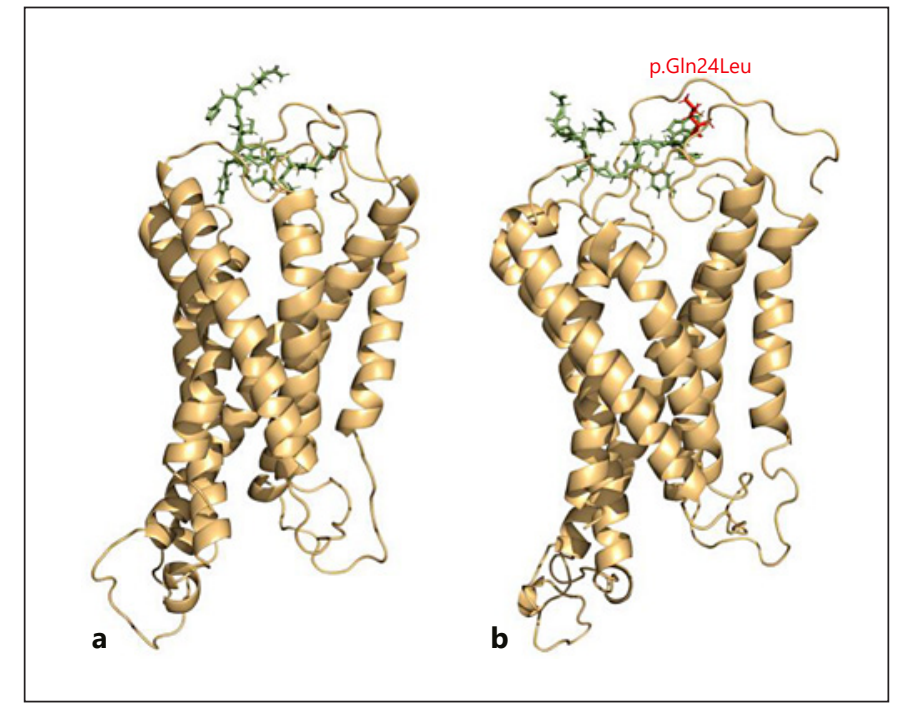

Fig. 2. The molecular dynamics simulation end structure (obtained after $100 \mathrm{~ns}$ ) represents atomic level interactions of GNRHR with GNRH-I (a) and GNRH-I (p.Gln24Leu) (b). The structure of GNRHR is shown in gold and GnRH-I is shown in green.

ture search. Ten $\mathrm{CHH}$ probands with biallelic GNRH1 variants (including 2 from the current study) have been reported to date. History of consanguinity was present in 6 , and family history with an affected sibling was reported in 4. Phenotype and genotype data were available for 13 patients ( 9 male probands and 4 females). Demographics, clinical findings, hormonal profile, pituitary imaging, and genotype of patients with biallelic GNRH1 variants are summarized in Table 1. The prevalence of biallelic GNRH1 variants in the $\mathrm{nCHH}$ cohorts ranges from 0.3 to $4 \%$. The geographical regions of origin of these patients were Turkey $(n=4)$, Armenia $(n=1)$, Romania $(n=1)$, South Asia $(n=3)$, and Brazil $(n=1)$. They presented with poor pubertal development at the median age of 16 (range: 13.2-25, IQR: 14.5-18) years. All patients had a severe reproductive phenotype. Cryptorchidism was present in $33 \%(3 / 9)$ of males. None had any nonreproductive features like hyposmia/anosmia, midline defects, or skeletal abnormalities except case $6 \mathrm{~b}$ who had a cleft palate. Serum FSH was $<1 \mathrm{mIU} / \mathrm{mL}$ in most $(12 / 13)$, whereas serum $\mathrm{LH}$ was $<1 \mathrm{mIU} / \mathrm{mL}$ in all (13/13). None of them had high serum prolactin (10/10). A total of seven biallelic pathogenic/likely pathogenic (P/LP) variants in GNRH1 were found in 10 probands. Of these, three were frameshift variants (p.Leu7ThrfsTer37, p.Leu30CysfsTer12, and p.Asp41GlufsTer8), two were missense (p.Arg31His and p.Gln24Leu), and two were splice-site variants (c.142$2 \mathrm{~A}>\mathrm{C}$ and c.238-2A $>\mathrm{G}$ ) (Fig. 3). All the reported biallelic 


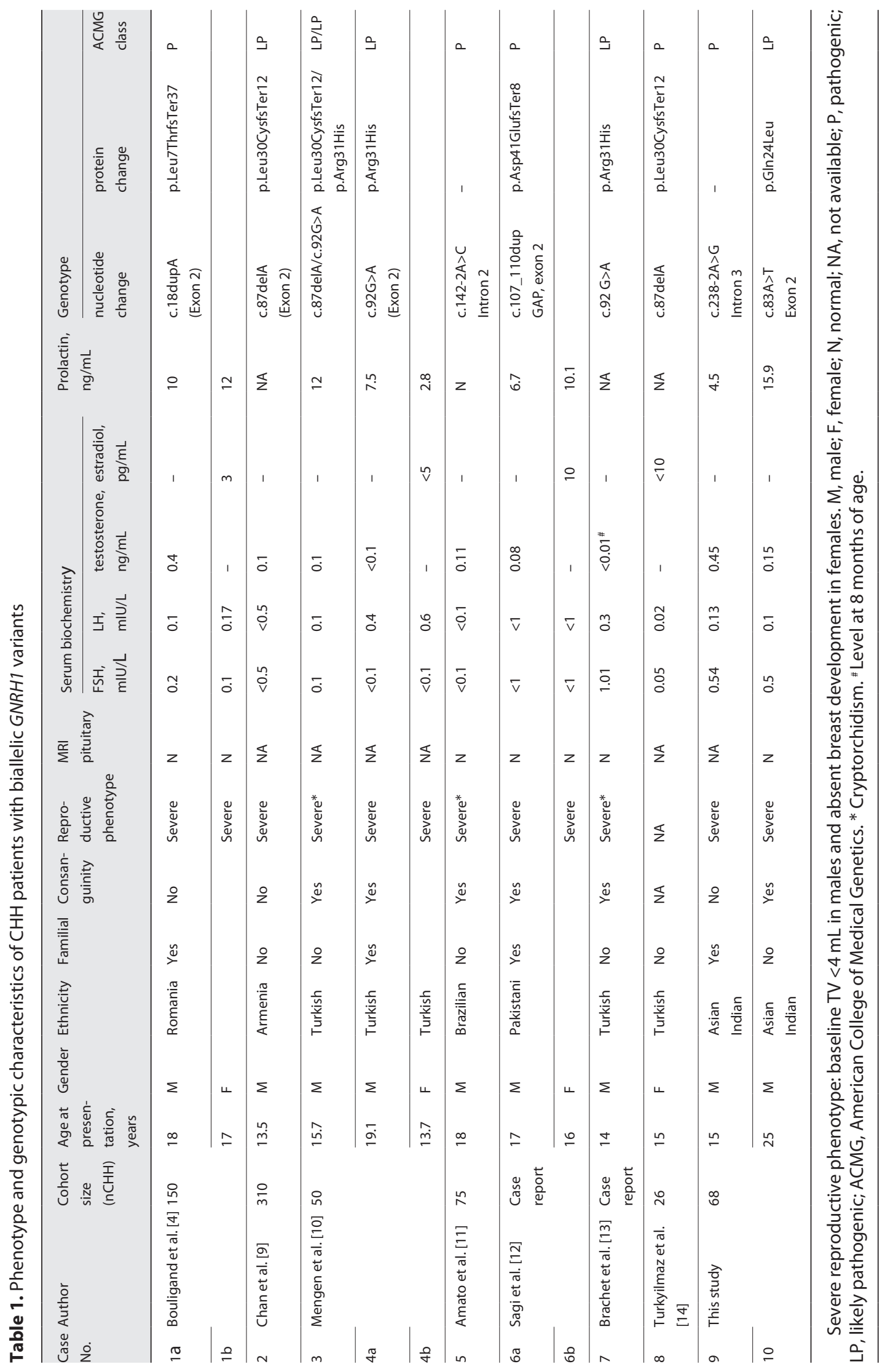




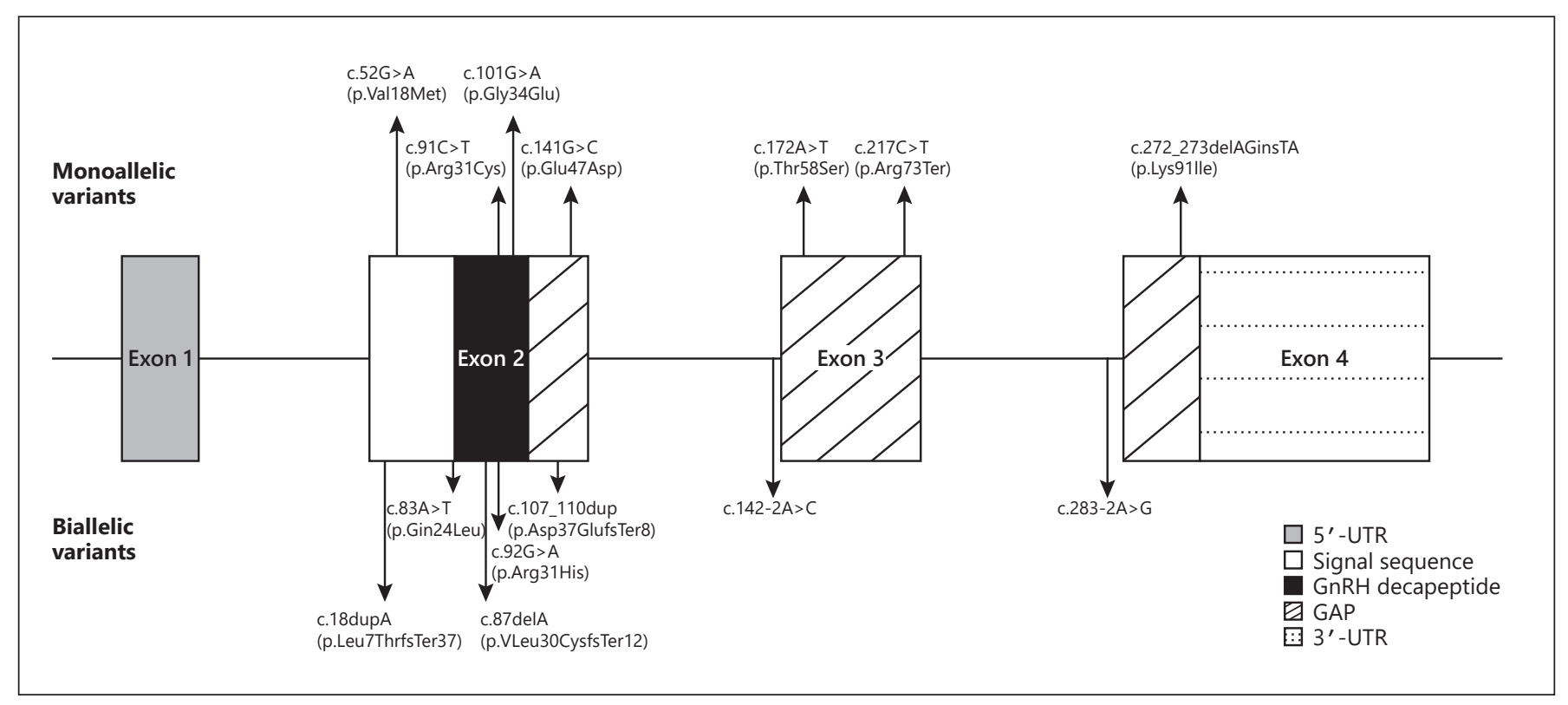

Fig. 3. Schematic diagram of the GNRH1 gene and distribution of reported variants.

variants are $\mathrm{P} / \mathrm{LP}$ as per ACMG classification criteria. All the five exonic variants were clustered in exon 2 , whereas intronic variants were observed in intron 2 and intron 3. Segregation data were available for all; parents with the variant in the heterozygous state had a normal reproductive function. p.Leu30CysfsTer12 and p.Arg31His were the two recurring variants reported from Turkey and Armenia. None had oligogenicity reported.

Characteristics of $11 \mathrm{CHH}$ probands (female: 3, male: 7, gender data not reported: 1) with seven GNRH1 monoallelic variants are shown in Table 2. The prevalence of monoallelic GNRH1 variants in the $\mathrm{CHH}$ cohorts varies from 0.5 to $2 \%$. Ethnic origin was Caucasian $(n=6)$, Asian $(n=2)$, or Latino $(n=1)$, whereas ethnicity data were not available in 2 cases. Five probands $(11,15,17$, 18 , and 19) had a severe reproductive phenotype, 2 had partial (probands 12 and13), whereas the data were not available in 4 cases. Serum FSH was $\geq 1 \mathrm{mIU} / \mathrm{mL}$ in all $(5 / 5)$, and serum $\mathrm{LH}$ was $\geq 1 \mathrm{mIU} / \mathrm{mL}$ in $3(3 / 5)$. Additionally, 4 probands also had nonreproductive phenotypes, hyposmia in 2 (probands 15 and 21), primary adrenal insufficiency in 1 (proband 19), and retinitis pigmentosa in 1 (proband 18). Amongst the seven variants, three (p.Glu47Asp, p.Thr58Ser, and p.Val18Met) were benign/likely benign (B/LB), two (p.Lys91Ile and p.Gly34Glu) were variants of unknown significance (VUS), and two (p.Arg31Cys, p.Arg73Ter) were P/LP. Two variants (p.Arg31Cys and p.Glu47Asp) were recurrent. Four probands (cases 11, 16, 19, and 20) exhibited oligogenicity with additional monoallelic variants [FGFR1 Ile239Thr (LP)/PROKR2 Ser202Gly (LB), FGFR1 c.6221G>T (P), FGFR1 splice-site (P), and DAX1 Pro167fsTer97 (P)] (Table 2). In 5 of the 7 probands (probands $11,12,13,14,17,19$, and 21) where family segregation data were available, the carrier parent harboring the heterozygous variant had a normal reproductive function. In two families, affected mothers (patient 11 and patient 13a, p.Arg31Cys) required assistance for reproduction, whereas 1 patient had a de novo variant (p.Arg31Cys).

\section{Discussion}

We describe 2 Asian Indian $\mathrm{CHH}$ probands with novel GNRH1 homozygous variants and report successful fertility with gonadotropin therapy in one. A systematic review of the reported cases with GNRH1 variants suggests GNRH1 biallelic variants lead to severe reproductive phenotype, with low gonadotropin levels. On the other hand, the role of reported GNRH1 monoallelic variants in the pathophysiology of $\mathrm{CHH}$ remains questionable.

The novel biallelic missense variant, p.Gln24Leu, alters the first amino acid (glutamine) of GnRH1 decapeptide, which gets converted to pyroglutamate (pGlu) posttranslationally. This amino acid (pGlu) is a highly conserved residue amongst vertebrates [8]. In rat studies, the 


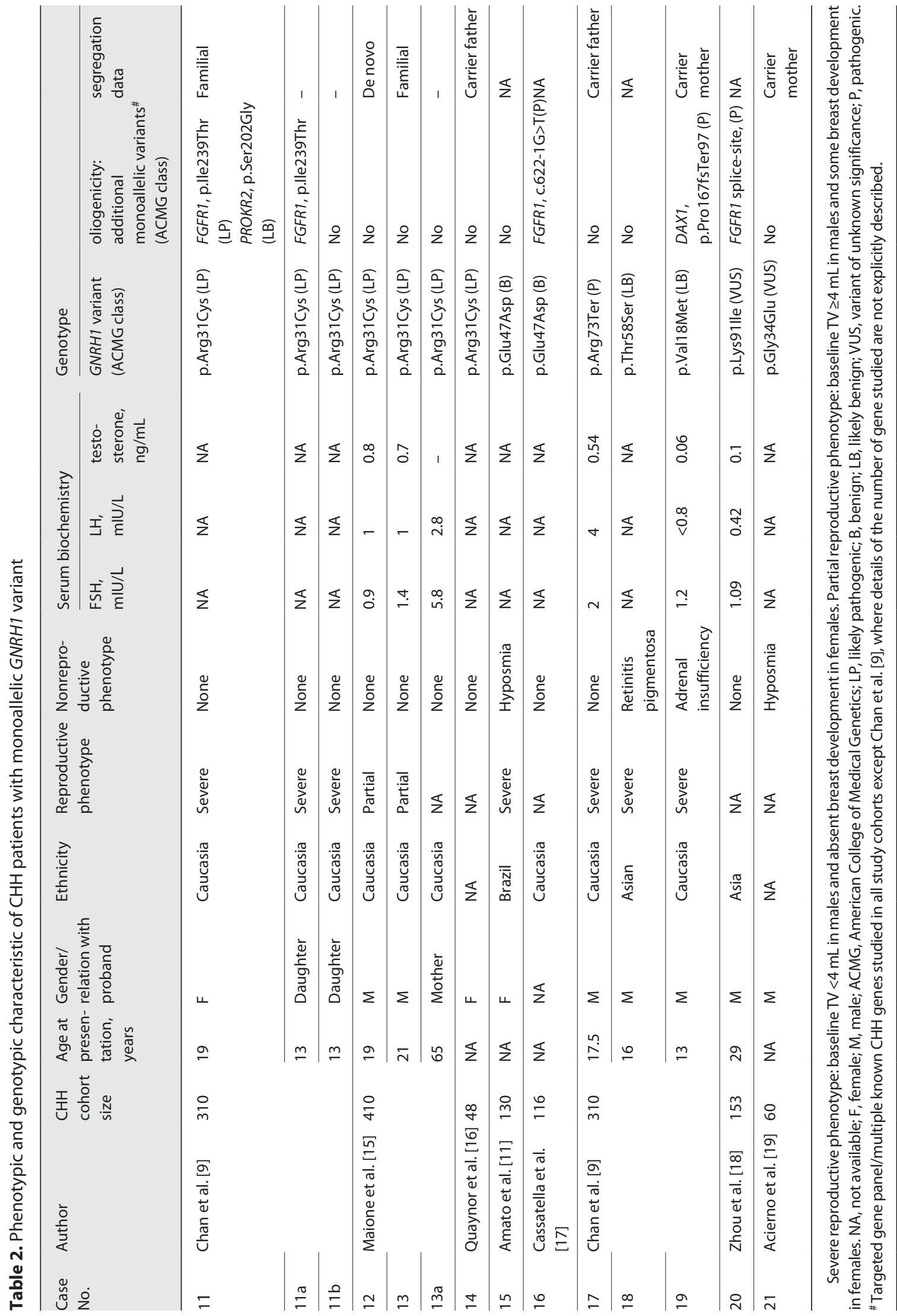


$\mathrm{GnRH}$ analog (with pGlu replaced by leucine) has very low potency $(0.003 \%)$ compared to the natural GnRH decapeptide [27] which was also confirmed in the MD simulation results from our study (Fig. 2). Our patient had an nCHH phenotype with low FSH, $\mathrm{LH}$, and T levels with a normal prolactin level. He responded to the gonadotrophin therapy, and fertility could be established after 18 months of treatment. Although previous reports mention sex steroid replacement therapy in $\mathrm{CHH}$ patients with biallelic GNRH1, this is the first case (case 10) of successful fertility with gonadotropin therapy in a $\mathrm{CHH}$ male with a biallelic GNRH1 variant.

Mutations in GNRH1 are a rare germline event, with very few patients reported worldwide. All the reported probands and their siblings harboring biallelic GNRH1 variants have a severe reproductive phenotype with low gonadotropin levels $(<1 \mathrm{mIU} / \mathrm{mL})$ and cryptorchidism in $3 / 8(37 \%)$ of the males (probands 3, 5, and 7). The severe phenotype suggests the reported pathogenic variants lead to poor GnRH activity. To date, seven biallelic pathogenic variants have been reported, of which two missense variants (p.Arg31His and p.Gln24Leu) occur in the $\mathrm{GnRH}$ decapeptide while another two variants, c.18dupA (p.Leu7ThrfsTer37) and c.87delA (p.Leu30CysfsTer12), cause frameshifts affecting the decapeptide and the GAP coding sequence. The GNRH1 variant, p.Leu7ThrfsTer37, is shown to have undetectable GnRH activity in AtT20 pituitary cells [4]. Notably, the rest three variants (p.Asp41GlufsTer8, c.142-2A >C, and c.238-2A $>$ G) affect only the GAP coding sequence without affecting GnRH decapeptide coding sequence and lead to a severe reproductive phenotype. Initial studies showed that GAP is an inhibitor of prolactin release [28]. Low/normal serum prolactin in all patients with biallelic GNRH1 variants questions the inhibitory role of GAP in prolactin regulation. The splice-site variant, c.142-2A $>C$, has been shown to cause aberrant splicing and could lead to loss of wildtype exons 3 and 4 [11]. In the lymphocytes of the patient with this splice-site variant, an aberrant GNRH1 transcript harboring a deletion of 4 bp (c.142-145delATAG) leading to a frameshift and a subsequent premature stop codon at amino acid position 74 was detected [11]. Another novel splice-site variant found in our patient, c.238$2 \mathrm{~A}>\mathrm{G}$, could lead to loss of exon 4 . How this alteration may lead to the $\mathrm{nCHH}$ phenotype is still unclear. A plausible explanation could be the loss of ESEs located on exon 4. These ESEs are purine-rich sequences that mediate posttranscriptional excision of the first intron of premRNA. These sequences are highly similar among mice, rats, and humans. In vitro (HeLa splicing system) and in vivo (hpg mouse) studies have shown that retention of intron 1 in the pre-mRNA affects translation of downstream open reading frame. The studies have also suggested that the splicing activity of ESE4 is much stronger than that of ESE3 $[6,7,29]$. Our novel intron 3 GNRH1 variant with $\mathrm{CHH}$ phenotype also suggests the role of exon 4 in pre-mRNA processing.

Haplotype analysis has proven the founder effect for the p.Leu7ThrfsTer37 variant described in Romanian probands [4]. Recurrent variants, p.Leu30CysfsTer12 and p.Arg31His, reported from the adjacent geographical region of Turkey and Armenia also suggest the founder effect for these variants. To date, only one biallelic missense variant, p.Arg31His, has been described in Turkish patients [10].

GNRH1 monoallelic variant, p.Arg31Cys, has been hypothesized to be responsible for the phenotype of the patients in multiple studies $[9,15,16]$. This variant is reported in many families and also from the geographically common areas. The other reported monoallelic variants (p.Glu47Asp, p.Thr58Ser, and p.Val18Met) were later classified as B/LB (Table 2, probands 15, 16, 18 , and 19) and were unlikely to be responsible for the pathogenesis of $\mathrm{CHH}$. A proband (Table 2, proband 21) carrying a monoallelic p.Gly34Glu (VUS) variant also had hyposmia. The Kallmann phenotype suggests the involvement of the GnRH neuronal development/migration pathway, rather than GnRH secretion. Consequently, the proband (Table 2, proband 20) carrying monoallelic variant, p.Lys91Ile (VUS), also harbored a pathogenic splice-site variant in FGFR1, which we believe can explain the $\mathrm{CHH}$ phenotype by itself. The presence of the heterozygous variant may be coincidental. A proband (Table 2, proband 17) carrying a nonsense heterozygous variant, p.Arg73Ter, had inherited it from his father who had a history of delayed puberty but normal reproduction. Conventionally delayed puberty is considered as a spectrum of $\mathrm{CHH}$, though recent genetic data suggest that constitutional delay in growth and puberty and $\mathrm{CHH}$ have distinct genetic profiles [17]. Even though the variant p.Arg31Cys is classified as LP and has been segregated positively in three unrelated families, in vitro studies have failed to confirm its dominant-negative effect [20]. Hence, the significance of the reported monoallelic variants remains unclear. Understanding the biological role of monoallelic variants needs further studies in transgenic animal models. The limitation of this systematic review includes a lack of phenotype and genetic segregation analysis data in all the $\mathrm{CHH}$ patients carrying monoallelic GNRH1 variants. 
To summarize, GNRH1 mutation as a cause of $\mathrm{CHH}$ is a rare event (1.3\% in the Asian Indian $\mathrm{CHH}$ cohort) suggesting a highly conserved gene structure with only a few cases described to date. We report two novel GNRH1 variants (missense and intronic) and successful gonadotropin-based therapy in 1 proband. The novel intron 3 variant in a human subject reiterates the role of exon 4 ESEs as previously shown in in vitro and in vivo studies. CHH patients with biallelic P/LP GNRH1 variants have severe reproductive phenotype with low gonadotropin levels, low/normal serum prolactin, and without nonreproductive phenotype, normal pituitary imaging, and low/normal serum prolactin. Amongst seven biallelic variants described to date, p.Leu30CysfsTer12 and p.Arg31His are recurring variants. The role of monoallelic $\mathrm{GnRH} 1$ variants in isolation in the pathogenesis of $\mathrm{CHH}$ remains questionable.

\section{Statement of Ethics}

The present study is a part of the research project No. EC/159/2009 entitled "Genotype, Phenotype and Radiological Correlation of Idiopathic Hypogonadotropic Hypogonadism," approved by the Institutional Ethics Committee II of Seth GS Medical College, Mumbai, India. Written informed consent was obtained from the study participants. Individual patient identity was kept confidential and coded before analysis.

\section{Conflict of Interest Statement}

The authors have no conflicts of interest to declare.

\section{Funding Sources}

The study was funded by the Ministry of Science and Technology, Department of Biotechnology, Government of India. There was neither any role of the funder in study design; collection, analysis, and interpretation of data; writing of the report; nor any restrictions regarding the submission of the report for publication.

\section{Author Contributions}

N.S., V.A.P., and A.R.L. conceived the idea and designed the study. V.A.P. and N.S. wrote the first draft of the report with input from A.R.L., S.A., and T.B. A.V.E. performed genetic study. V.S., R.S., and S.S.M. accessed and verified the data. S.S.M. performed the statistical analysis which was verified by V.A.P. and V.S. V.V.B. and A.K. studied the molecular model. V.A.P., R.S., A.R.L., N.S., and T.B. were involved in management of patients and data collection. V.S., S.S.M., S.A., and T.B. supervised entire data collection and management and provided inputs in revision of the draft. All the authors were involved in critical revision of the manuscript.

\section{Data Availability Statement}

The data that support the findings of this study are available from the corresponding author (A.R.L.) upon reasonable request.

\section{References}

1 Butz H, Nyírő G, Kurucz PA, Likó I, Patócs A. Molecular genetic diagnostics of hypogonadotropic hypogonadism: from panel design towards result interpretation in clinical practice. Hum Genet. 2021 Jan;140(1):113-34

2 Mason A, Hayflick J, Zoeller R, Young W, Phillips $\mathrm{H}$, Nikolics K, et al. A deletion truncating the gonadotropin-releasing hormone gene is responsible for hypogonadism in the hpg mouse. Science. 1986 Dec;234(4782): 1366-71.

3 Mason A, Pitts S, Nikolics K, Szonyi E, Wilcox J, Seeburg P, et al. The hypogonadal mouse: reproductive functions restored by gene therapy. Science. 1986 Dec;234(4782):1372.

4 Bouligand J, Ghervan C, Tello JA, BraillyTabard S, Salenave S, Chanson P, et al. Isolated familial hypogonadotropic hypogonadism and a GNRH1 mutation. N Engl J Med. 2009 Jun;360(26):2742-8.

5 Cheng CK, Leung PC. Molecular biology of Gonadotropin-Releasing Hormone (GnRH)I, GnRH-II, and their receptors in humans. Endocr Rev. 2005 Apr;26(2):283-306.
6 Han J, Seong JY, Kim K, Wuttke W, Jarry H. Analysis of exonic splicing enhancers in the mouse gonadotropin-releasing hormone $(\mathrm{GnRH})$ gene. Mol Cell Endocrinol. $2001 \mathrm{Feb}$; 173(1-2):157-66.

7 Seong JY, Park S, Kim K. Enhanced splicing of the first intron from the Gonadotropin-Releasing Hormone $(\mathrm{GnRH})$ primary transcript is a prerequisite for mature $\mathrm{GnRH}$ messenger RNA: presence of $\mathrm{GnRH}$ neuron-specific splicing factors. Mol Endocrinol. 1999;13(11): 1882-95.

8 Okubo K, Nagahama Y. Structural and functional evolution of gonadotropin-releasing hormone in vertebrates. Acta Physiol. 2008 May;193(1):3-15.

9 Chan Y-M, de Guillebon A, Lang-Muritano M, Plummer L, Cerrato F, Tsiaras S, et al. GNRH1 mutations in patients with idiopathic hypogonadotropic hypogonadism. Proc Natl Acad Sci U S A. 2009 Jul;106(28):117038.

10 Mengen E, Tunc S, Kotan LD, Nalbantoglu O, Demir K, Gurbuz F, et al. Complete idiopath- ic hypogonadotropic hypogonadism due to homozygous GNRH1 mutations in the mutational hot spots in the region encoding the decapeptide. Horm Res Paediatr. 2016;85(2): 107-11.

11 Amato LGL, Montenegro LR, Lerario AM, Jorge AAL, Guerra Junior G, Schnoll C, et al. New genetic findings in a large cohort of congenital hypogonadotropic hypogonadism. Eur J Endocrinol. 2019 Aug;181(2):10319.

12 Sagi SV, Joshi H, Whiles E, Hikmat M, Puthi VR, MacDougall J, et al. Normosmic idiopathic hypogonadotropic hypogonadism due to a novel GNRH1 variant in two siblings. Endocrinol Diabetes Metab Case Rep. 2020 Mar; 2020. Epub ahead of print.

13 Brachet C, Gernay C, Boros E, Soblet J, Vilain C, Heinrichs C. Homozygous p.R31H GNRH1 mutation and normosmic congenital hypogonadotropic hypogonadism in a patient and self-limited delayed puberty in his relatives. J Pediatr Endocrinol Metab. 2020 Sep;33(9):1237-40. 
14 Turkyilmaz A, Cayir A, Yarali O, Kurnaz E, Kartal Baykan E, Arslan Ates E, et al. Clinical characteristics and molecular genetic analysis of a cohort with idiopathic congenital hypogonadism. J Pediatr Endocrinol Metab. 2021 Apr;34(6):771-80.

15 Maione L, Albarel F, Bouchard P, Gallant M, Flanagan CA, Bobe R, et al. R31C GNRH1 mutation and congenital hypogonadotropic hypogonadism. PLoS One. 2013 Jul;8(7): e69616.

16 Quaynor SD, Kim H-G, Cappello EM, Williams T, Chorich LP, Bick DP, et al. The prevalence of digenic mutations in patients with normosmic hypogonadotropic hypogonadism and Kallmann syndrome. Fertil Steril. 2011 Dec;96(6):1424-30.e6.

17 Cassatella D, Howard SR, Acierno JS, Xu C, Papadakis GE, Santoni FA, et al. Congenital hypogonadotropic hypogonadism and constitutional delay of growth and puberty have distinct genetic architectures. Eur J Endocrinol. 2018 Apr;178(4):377-88.

18 Zhou C, Niu Y, Xu H, Li Z, Wang T, Yang W, et al. Mutation profiles and clinical characteristics of Chinese males with isolated hypogonadotropic hypogonadism. Fertil Steril. 2018 Aug;110(3):486-95.e5.
19 Acierno JS, Xu C, Papadakis GE, Niederländer NJ, Rademaker JD, Meylan J, et al. Pathogenic mosaic variants in congenital hypogonadotropic hypogonadism. Genet Med. 2020 Nov;22(11):1759-67.

20 Maione L, Albarel F, Bouchard P, Gallant M, Flanagan CA, Bobe R, et al. R31C GNRH1 mutation and congenital hypogonadotropic hypogonadism. PLoS One. 2013 Jul;8(7): e69616.

21 Nair S, Jadhav S, Lila A, Jagtap V, Bukan A, Pandit R, et al. Spectrum of phenotype and genotype of congenital isolated hypogonadotropic hypogonadism in Asian Indians. Clin Endocrinol. 2016 Jul;85(1):100-9.

22 Young J, Xu C, Papadakis GE, Acierno JS, Maione L, Hietamäki J, et al. Clinical management of congenital hypogonadotropic hypogonadism. Endocr Rev. 2019 Apr;40(2):669710 .

23 Arya S, Tiwari A, Lila AR, Sarathi V, Bhandare VV, Kumbhar BV, et al. Homozygous p.Val89Leu plays an important pathogenic role in $5 \alpha$-reductase type 2 deficiency patients with homozygous p.Arg246Gln in SRD5A2. Eur J Endocrinol. 2020 Sep;183(3):275-84.
24 Page MJ, McKenzie JE, Bossuyt PM, Boutron I, Hoffmann TC, Mulrow CD, et al. The PRISMA 2020 statement: an updated guideline for reporting systematic reviews. PLoS Med. 2021 Mar;18(3):e1003583.

25 VarSome. VarSome citations [Internet]. 2020 Jan [cited 2021 May 15]. Available from: https: //varsome.com/about/general/varsome-citations/.

26 Richards S, Aziz N, Bale S, Bick D, Das S, Gastier-Foster J, et al. Standards and guidelines for the interpretation of sequence variants: a joint consensus recommendation of the American College of Medical Genetics and Genomics and the Association for Molecular Pathology. Genet Med. 2015 May; 17(5):405-23.

27 Coy DH, Coy EJ, Schally AV. Effect of simple amino acid replacements on the biological activity of luteinizing hormone-releasing hormone. J Med Chem. 1973 Oct;16(10):1140-3.

28 Chan Y-M. A needle in a haystack: mutations in GNRH1 as a rare cause of isolated $\mathrm{GnRH}$ deficiency. Mol Cell Endocrinol. 2011 Oct; 346(1-2):51-6.

29 Son GH, Jung H, Seong JY, Choe Y, Geum D, Kim K. Excision of the first intron from the Gonadotropin-releasing Hormone (GnRH) transcript serves as a key regulatory step for GnRH biosynthesis. J Biol Chem. 2003 May; 278(20):18037-44. 